\title{
Time evolution of an anharmonic oscillator interacting with a squeezed bath
}

\author{
G. M. D'Ariand \\ Dipartimento di Fisica "Alessandro Volta", Università di Pavia, Via A. Bassi 6, I-27100 Pavia, Italy \\ M. Fortunatd \\ Dipartimento di Fisica, Università di Roma "La Sapienza", P.le A. Moro 2, I-00185 Roma, Italy \\ and \\ P. Tombesit \\ Dipartimento di Matematica e Fisica, Università di Camerino, Via Madonna delle Carceri, I-62032 Camerino, Italy
}

(Received August 15, 2018)

The evolution of a single mode of the electromagnetic field interacting with a squeezed bath in a Kerr medium is considered. The solution of the corresponding master equation is given numerically. It is argued that the creation of a superposition state (Schrödinger's cat) is better achieved in presence of a squeezed reservoir than of a thermal one.

PACS number: 42.50.Dv

\section{INTRODUCTION}

The possibility of detecting quantum interference effects between macroscopically distinguishable stateseigenstates of a variable corresponding to macroscopically different eigenvalues - has received much attention in the last few years [1] 10 . Such interest is motivated by one of the fundamental problems in quantum mechanics: to determine whether quantum-mechanical features can be observed in macroscopic objects [11. Unfortunately, the detection of a macroscopic superposition is very difficult, due to the unavoidable coupling with the environment and by the consequent dissipation 12 . The severity with which dissipation destroys the quantum coherence at macroscopic level has been discussed by several authors 13 19].

Since the original proposal by Yurke and Stoler [1], who have shown that a coherent state propagating through a Kerr medium evolves, under suitable conditions, into a quantum superposition of two coherent states which are $180^{\circ}$ out of phase with each other, many attempts have been made in order to preserve this superposition with detectable effects as, for example, the interference fringes at the output of a homodyne detector. Mecozzi and Tombesi [3 5] have considered a dissipation model corresponding to a beam splitter with a squeezed vacuum injected into the unused port. They have shown that the interference pattern may be preserved if the input light is squeezed in a suitable quadrature. As a model for phase-sensitive measurements, Kennedy and Walls [6] suggested the use of a squeezed bath in place of the thermal one, showing a substantial improvement of the quadrature-phase sensitivity. On these lines, Bužek, Kim and Gantsog [10] have recently studied the phase properties of superpositions of coherent states evolving in a squeezed (phase-sensitive) amplifier, showing that differently from the phase-insensitive case [19], the phase distribution of the Schrödinger-cat input state can be preserved for long times.

The above models either suffer a parametrically imposed dynamics for the loss (beam splitter models [1.3. 51), or do not consider the nonlinear coupling generating the superposition state, which is just treated as an initial condition 6, 10]. For this reason we consider a model in which both the anharmonic Hamiltonian and the interaction with a squeezed bath are taken into account, thus allowing a test of the effect of squeezed fluctuations on the generation of a superposition state starting from a single coherent one.

This article is organized as follows: in Sec. II we introduce the model and discuss the corresponding master equation. In Sec. III we present the numerical integration of the master equation and the results of the numerical analysis, showing the efficiency of a squeezed bath in the creation of a "quasi-superposition state". In Sec. IV we conclude and summarize the results.

\section{THE MODEL AND THE MASTER EQUATION}

We consider a mode of the electromagnetic field at frequency $\omega$ in a Kerr medium coupled to a squeezed bath, namely a reservoir of oscillators whose fluctuations are squeezed [6]. The total Hamiltonian is given by

$$
H=H_{\mathrm{S}}+H_{\mathrm{I}}+H_{\mathrm{B}},
$$

where $H_{\mathrm{S}}$ is the free Kerr Hamiltonian of the field mode 


$$
H_{\mathrm{S}}=\omega\left(a^{\dagger} a\right)+\Omega\left(a^{\dagger} a\right)^{2},
$$

$H_{\mathrm{B}}$ is the free Hamiltonian of the bath, and $H_{\mathrm{I}}$ is the oscillator-reservoir interaction Hamiltonian, which in the rotating wave approximation has the form

$$
H_{\mathrm{I}}=a^{\dagger} \hat{\Gamma}+a \hat{\Gamma}^{\dagger} .
$$

In Eqs. (2) and (3) $a^{\dagger}$ and $a$ are the boson creation and annihilation operators of the mode, while $\hat{\Gamma}^{\dagger}$ and $\hat{\Gamma}$ are bath operators. The bath is squeezed and Markovian, namely the correlations functions of the operators $\hat{\Gamma}^{\dagger}$ and $\hat{\Gamma}$ are given by [20]

$$
\begin{aligned}
\left\langle\hat{\Gamma}^{\dagger}(t) \hat{\Gamma}\left(t^{\prime}\right)\right\rangle & =2 \gamma N \delta\left(t-t^{\prime}\right), \\
\left\langle\hat{\Gamma}(t) \hat{\Gamma}^{\dagger}\left(t^{\prime}\right)\right\rangle & =2 \gamma(N+1) \delta\left(t-t^{\prime}\right), \\
\left\langle\hat{\Gamma}(t) \hat{\Gamma}\left(t^{\prime}\right)\right\rangle & =2 \gamma M e^{-2 i \omega t} \delta\left(t-t^{\prime}\right), \\
\left\langle\hat{\Gamma}^{\dagger}(t) \hat{\Gamma}^{\dagger}\left(t^{\prime}\right)\right\rangle & =2 \gamma M^{*} e^{2 i \omega t} \delta\left(t-t^{\prime}\right) .
\end{aligned}
$$

In Eqs. (4) $\gamma$ is the damping constant (determined by the coupling between the oscillator and the bath), $N$ is a real parameter, which reduces to the mean number of thermal photons when the bath is not squeezed, and $M$ is the squeezing complex parameter $\left(M=|M| e^{i \psi}\right)$ satisfying the relation

$$
|M| \leq \sqrt{N(N+1)} .
$$

The parameters $N$ and $M$ measure the strength of the correlations of the bath degrees of freedom: for $|M|^{2}=$ $N(N+1)$ the squeezing is maximum, whereas for $|M|=0$ the reservoir reduces to the customary thermal one.

From Eqs. (11)-(4) one can derive the following master equation for the reduced density matrix of the field mode in the interaction picture 20

$$
\begin{aligned}
\frac{d \hat{\rho}}{d t}= & -i \Omega\left[\left(a^{\dagger} a\right)^{2}, \hat{\rho}\right]+\gamma(N+1)\left(2 a \hat{\rho} a^{\dagger}-a^{\dagger} a \hat{\rho}-\hat{\rho} a^{\dagger} a\right) \\
& +\gamma N\left(2 a^{\dagger} \hat{\rho} a-a a^{\dagger} \hat{\rho}-\hat{\rho} a a^{\dagger}\right) \\
& -\gamma M\left(2 a^{\dagger} \hat{\rho} a^{\dagger}-a^{\dagger} a^{\dagger} \hat{\rho}-\hat{\rho} a^{\dagger} a^{\dagger}\right) \\
& -\gamma M^{*}(2 a \hat{\rho} a-a a \hat{\rho}-\hat{\rho} a a) .
\end{aligned}
$$

Eq. (5) is numerically solved in the next section. The results are shown in terms of the $Q$-function

$$
Q\left(\alpha, \alpha^{*}, t\right)=\langle\alpha|\hat{\rho}(t)| \alpha\rangle,
$$

which is the (positive definite) probability density for the antinormally ordered moments of the annihilation and creation operators, and in terms of the Wigner function, which is defined by

$$
W\left(\alpha, \alpha^{*}, t\right)=\int \frac{d^{2} \lambda}{\pi^{2}} e^{-\lambda \alpha^{*}+\lambda^{*} \alpha} \operatorname{Tr}\left\{\hat{\rho}(t) e^{\lambda a^{\dagger}-\lambda^{*} a}\right\} .
$$

Using standard methods [13], it is possible to convert the master equation (6) into the Fokker-Planck type equation for the $Q$-function

$$
\begin{aligned}
\frac{\partial Q}{\partial t}= & -i \Omega\left[\alpha^{*}\left(1+2|\alpha|^{2}\right) \frac{\partial Q}{\partial \alpha^{*}}-\alpha\left(1+2|\alpha|^{2}\right) \frac{\partial Q}{\partial \alpha}\right. \\
& \left.+\left(\alpha^{*}\right)^{2} \frac{\partial^{2} Q}{\partial \alpha^{* 2}}-\alpha^{2} \frac{\partial^{2} Q}{\partial \alpha^{2}}\right] \\
& +\gamma\left(\frac{\partial}{\partial \alpha} \alpha+\frac{\partial}{\partial \alpha^{*}} \alpha^{*}\right) Q+2 \gamma(N+1) \frac{\partial^{2} Q}{\partial \alpha \partial \alpha^{*}} \\
& +\gamma M \frac{\partial^{2} Q}{\partial \alpha^{2}}+\gamma M^{*} \frac{\partial^{2} Q}{\partial \alpha^{* 2}} .
\end{aligned}
$$

Eq. (9) has been solved for $\Omega=0$ [6] and for $M=0$ [19]. For $\Omega$ and $M$ both nonvanishing, however, an analytic solution is not available, whereas numerical integration has to be carried out carefully, because of computation instabilities 21.

In absence of dissipation an initial coherent state

$$
\left|\alpha_{0}\right\rangle=\exp \left(-\frac{\left|\alpha_{0}\right|^{2}}{2}\right) \sum_{n=0}^{\infty} \frac{\alpha_{0}^{n}}{\sqrt{n !}}|n\rangle
$$

evolves towards the following superposition of coherent states at $t=\pi / 2 \Omega$

$$
|\phi\rangle=\frac{1}{\sqrt{2}}\left(e^{-i \pi / 4}\left|\alpha_{0}\right\rangle+e^{i \pi / 4}\left|-\alpha_{0}\right\rangle\right) .
$$

The $Q$-function of the $t=0$ coherent state is the Gaussian

$$
Q\left(\alpha, \alpha^{*}, 0\right)=\exp \left(-\left|\alpha-\alpha_{0}\right|^{2}\right),
$$

whereas for $t=\pi / 2 \Omega$ it approximatively corresponds to two Gaussian peaks in the complex plane centered at $\alpha_{0}$ and $-\alpha_{0}$. The two peaks are "macroscopically distinguishable" for $\left|\alpha_{0}\right| \gg 1$. The evolution is periodic with period $T=2 \pi / \Omega$. In presence of dissipation the state at times $t>0$ is no longer pure, and a superposition of states corresponds to a "quasi-superposition" mixed state, with interfering features still surviving. We will look for signatures of such states from peaks in the complex plane approximately separated by a distance $2\left|\alpha_{0}\right|$, reminiscent of the state (11).

\section{NUMERICAL RESULTS}

We have studied the time evolution of the density matrix by integrating numerically the master equation (6) for truncated Hilbert space dimension $d=128$ (typically a power of 2, in order to take advantage of fast Fourier transform algorithm for reconstructing the $Q$-function and the Wigner function). The integration time-step has to be carefully tuned as a function of $\Omega$ and $\alpha_{0}$ (here typically $\gamma \Delta t \simeq 10^{-5}$ for a standard fourth-order RungeKutta routine). Numerical accuracy is checked through normalization of $\hat{\rho}, Q$ and $W$, positivity of $Q$, and reality of the diagonal elements of $\hat{\rho}$. As a test, the results from analytical solutions for $\gamma=0$ [1,2] and for $\Omega=0$ [6] have been recovered up to the seventh digit. 
In Fig. 11 the $Q$-function from the master equation (6) for maximally squeezed bath $[|M|=\sqrt{N(N+1)}]$ is given for $\Omega / \gamma=10, N=30$, and squeezing phase $\psi=0$. At $\gamma t=0.0576$ [well before $\Omega t=\pi / 2$, namely before the creation time of the state (11) in the undamped case] two peaks are clearly visible in the structure of the $Q$-function. This result can be compared with that of Daniel and Milburn [19] at the same time, but in absence of squeezing. The two peaks are almost symmetrical with respect to the origin of the phase space. Of course, due to dissipation, they are not strictly coherent, but they are still visible, even for subsequent times.

In Fig. 2 the effect of a purely thermal bath on the generation of the superposition state is shown for comparison with Fig. 1 for the same values of the parameters. In this case there are no peaks in the $Q$-function, which now exhibits a classical behaviour [17]: this is the usual effect of dissipation [14].

In Fig. 3 we show for completeness the time evolution of the Wigner function at the same times as in Fig. 1: the two peaks still show up, but now some interference features arise between the two component states, as expected. We have also considered the case in which the phase of the complex squeezing parameter $M$ is chosen as $\psi=\pi$, namely the squeezing direction of the phase sensitive bath is rotated of $90^{\circ}$ with respect to the case $\psi=0$ 10,22 : the creation of two peaks is still visible and their distance is approximately the same as in Fig. 1, but the line connecting them is now rotated of about $90^{\circ}$, in agreement with the phase of $M$.

The effect is further confirmed by the behaviour of the quadrature distributions $P\left(x_{\phi}\right)$, defined by

$$
P\left(x_{\phi}\right)=\left\langle x_{\phi}|\hat{\rho}| x_{\phi}\right\rangle
$$

with

$$
\hat{x}_{\phi}=\frac{1}{\sqrt{2}}\left(e^{i \phi} a+e^{-i \phi} a^{\dagger}\right) .
$$

The probability $P\left(x_{\phi}\right)$ is plotted in Fig. A at $\gamma t=0.0576$ for $\phi=\pi / 4$ and $\phi=3 \pi / 4$. For $\phi=3 \pi / 4$-along the direction which joins the two peaks of the Wigner function - the probability $P\left(x_{\phi}\right)$ exhibits two peaks resulting from marginal integration in the complex plane. On the other hand, along the orthogonal direction at $\phi=$ $\pi / 4$, a pattern reminiscent of interference in phase space 23] between the two originally coherent components is visible. We have also computed the degree of mixing $S=1-\operatorname{Tr}\left(\hat{\rho}^{2}\right)$ : its behaviour shows a rapid increase with time, achieving the asymptotic value $S=0.96$. Thus, the above coherence features still survive notwithstanding the large mixing. Notice that, however, this happens only for the two-peak state, whereas there is no reminiscence of the symmetrical superposition of three coherent states which arises at $t=\pi / 3 \Omega$ in absence of dissipation.

As regards the other parameters, the overall scenario is not affected by changing the phase of the initial coherent field $\alpha_{0}$, whereas increasing the modulus of $\alpha_{0}$ deteriorates the formation of the two peaks. On the other hand, slightly increasing $\Omega$ or $N$ (still for a maximally squeezed bath), does not appreciably improve the visibility of the peaks.

\section{SUMMARY AND CONCLUSIONS}

We have considered the anharmonic oscillator interacting with a squeezed bath. The dissipative Kerr effect models a coherent state propagating into a nonlinear medium, as, for example, into an optical fiber. The squeezing has been considered in order to have the production of coherent effects in presence of dissipation. Differently from the previous works [6] and [10], our model treats all effects (nonlinearity, dissipation and squeezing) contextually. We have shown that squeezing the fluctuations of the bath improves the generation of "quasisuperposition" states, which are completely forbidden in presence of a heat bath. Our results are in agreement with those of Kennedy and Walls 6 and of Bužek, Kim and Gantsog 10 and complete them: a squeezed bath not only is able to preserve a macroscopic quantum superposition, but also allows its generation in presence of nonlinear interactions. The physical meaning of a squeezed bath is not completely understood in the present context: a suitable feedback mechanism could be envisaged that supports an ad hoc phase sensitive interaction, which amplifies or attenuates fluctuations, depending on the quadrature 24].

In conclusion, some comments regarding the direction of squeezing in the bath are in order. Despite the squeezing direction does not affects the generation of quasisuperposition states, the survival time of coherence is naturally increased only for squeezing in the direction orthogonal to the line that joins the component states. On the other hand, the Kerr effect rotates the $Q$-function in the complex plane, thus making squeezing less efficient in the overall evolution. This suggests that an ideal bath should be squeezed isotropically in the plane, depending on the phase of the state itself. It is possible to write a master equation for such an isotropically squeezed bath: numerical results on these lines will be the object of a forthcoming paper 25].

We would like to thank Prof. M. Cini for many stimulating and helpful discussions on this topic.

* E-mail: dariano@vaxpv.pv.infn.it

$\dagger$ E-mail: fortunato@vaxrom.roma1.infn.it

¥ E-mail: tombesi@vaxrom.roma1.infn.it

[1] B. Yurke and D. Stoler, Phys. Rev. Lett. 57, 13 (1986).

[2] G. J. Milburn, Phys. Rev. A 33, 674 (1986). 
[3] A. Mecozzi and P. Tombesi, Phys. Rev. Lett. 58, 1055 (1987).

[4] P. Tombesi and A. Mecozzi, J. Opt. Soc. Am. B 4, 1700 (1987).

[5] A. Mecozzi and P. Tombesi, Phys. Lett. A 121, 101 (1987).

[6] T. A. B. Kennedy and D. F. Walls, Phys. Rev. A 37, 152 (1988).

[7] G. J. Milburn, A. Mecozzi and P. Tombesi, J. Mod. Opt. 36, 1607 (1989).

[8] G. J. Milburn, Phys. Rev. A 39, 2749 (1989).

[9] M. Brisudová, J. Mod. Opt. 38, 2505 (1991).

[10] V. Bužek, M. S. Kim and Ts. Gantsog, Phys. Rev. A 48, 3394 (1993).

[11] A. J. Leggett, Prog. Theor. Phys. Suppl. 69, 80 (1980); Lesson of Quantum Theory, Niels Bohr Centenary Symposium, pp. 35-37 (Ed. de Boer, 1985).

[12] W. H. Zurek, Phys. Rev. D 24, 1516 (1981); Phys. Rev. D 26, 1862 (1982).

[13] G. J. Milburn and D. F. Walls, Am. J. Phys. 51, 1134 (1983).

[14] A. O. Caldeira and A. J. Leggett, Phys. Rev. A 31, 1059 (1985).

[15] D. F. Walls and G. J. Milburn, Phys. Rev. A 31, 2403 (1985).

[16] C. M. Savage and D. F. Walls, Phys. Rev. A 32, 2316 (1985).

[17] G. J. Milburn and C. A. Holmes, Phys. Rev. Lett. 56, 2237 (1986).

[18] G. J. Milburn and D. F. Walls, Phys. Rev. A 38, 1087 (1988).

[19] D. J. Daniel and G. J. Milburn, Phys. Rev. A 39, 4628 (1989).

[20] C. W. Gardiner, Quantum Noise (Springer-Verlag, Berlin, 1991).

[21] L. Collatz, The Numerical Treatment of Differential Equations (Springer-Verlag, Berlin, 1966).

[22] R. Loudon and P.L. Knight, J. Mod. Opt. 34, 709 (1987).

[23] W. Schleich and J. A. Wheeler, Nature (London) 326, 574 (1986); W. Schleich and J. A. Wheeler, J. Opt. Soc. Am. B 4, 1715 (1987).

[24] P. Tombesi and D. Vitali, private communication.

[25] G. M. D'Ariano, M. Fortunato and P. Tombesi (unpublished).

FIG. 1. Contour plots of the time evolved $Q$-function according to Eq. (6). Here $N=30,|M|=\sqrt{N(N+1)}, \psi=0$, $\alpha_{0}=3$, and $\Omega / \gamma=10$.

FIG. 2. The same as in Fig. 1, but for $M=0$.

FIG. 3. The Wigner function corresponding to plots in Fig. 11.

FIG. 4. Plot of the marginal distribution $P\left(x_{\phi}\right)$ at $\gamma t=0.0576$ for $\phi=3 \pi / 4$ and $\phi=\pi / 4$. 

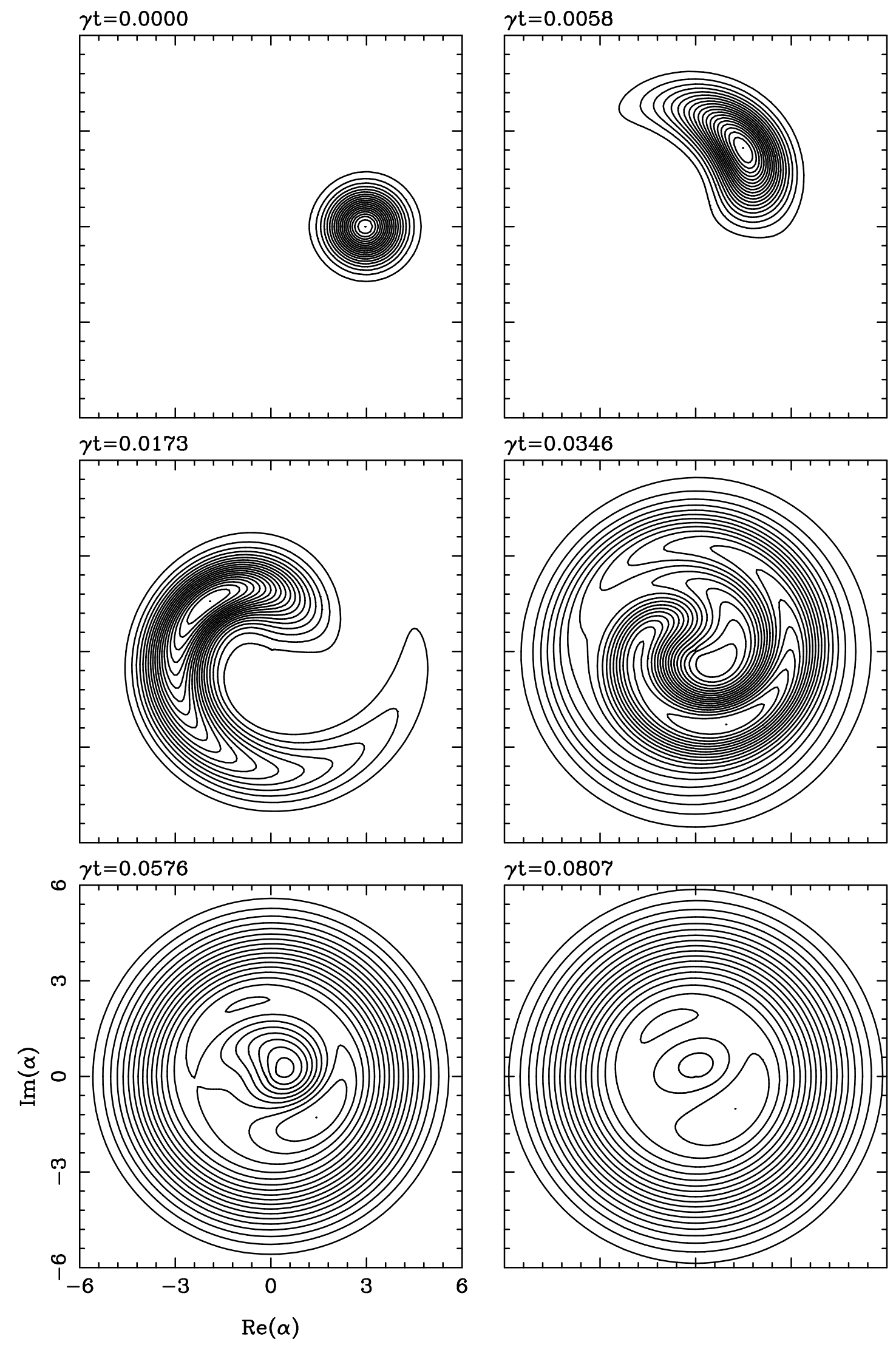

Fig.1 G. M. D'Ariano, M. Fortunato, and P. Tombesi Time evolution of an anharmonic oscillator 

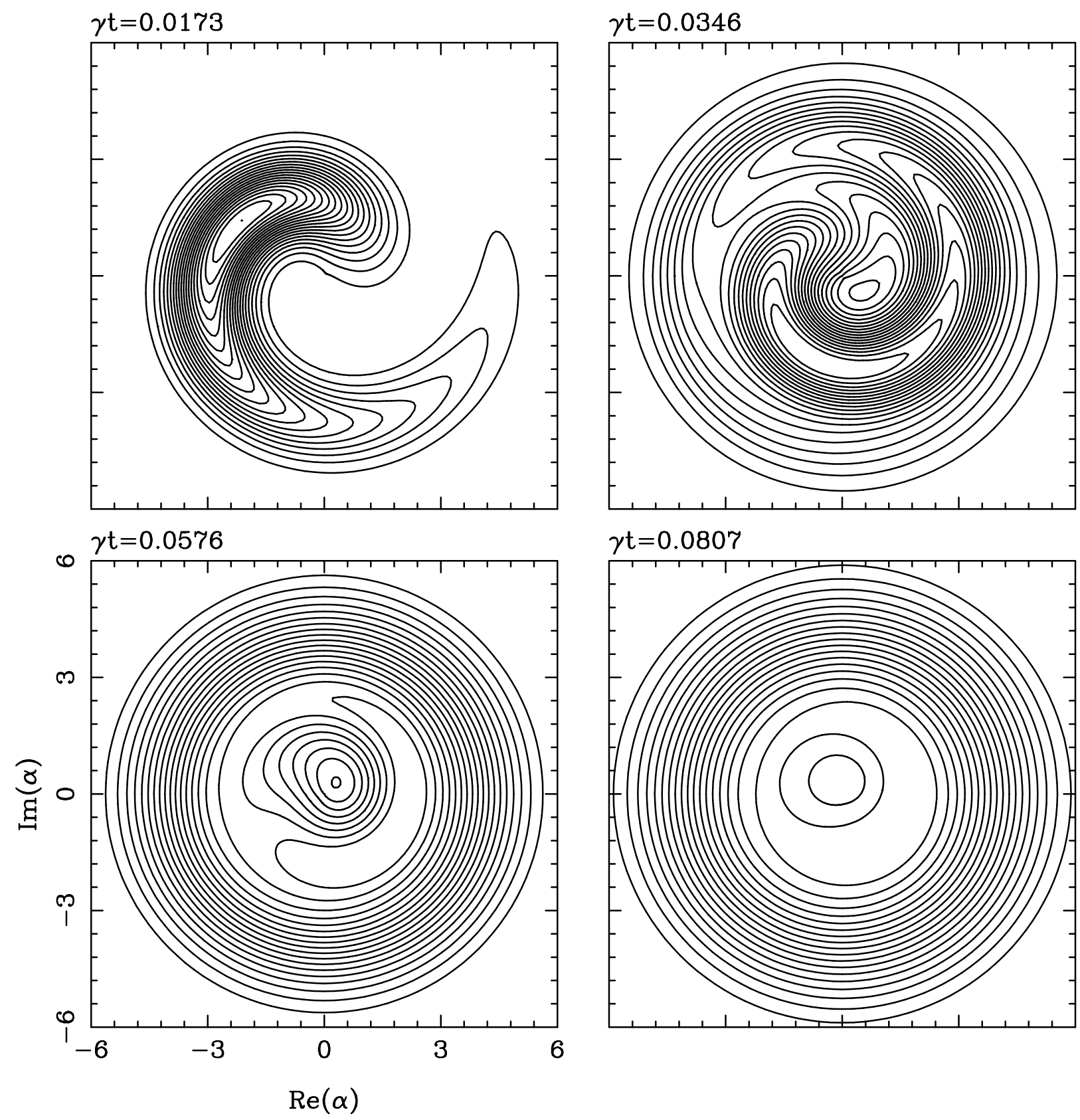

Fig.2 G. M. D'Ariano, M. Fortunato, and P. Tombesi 

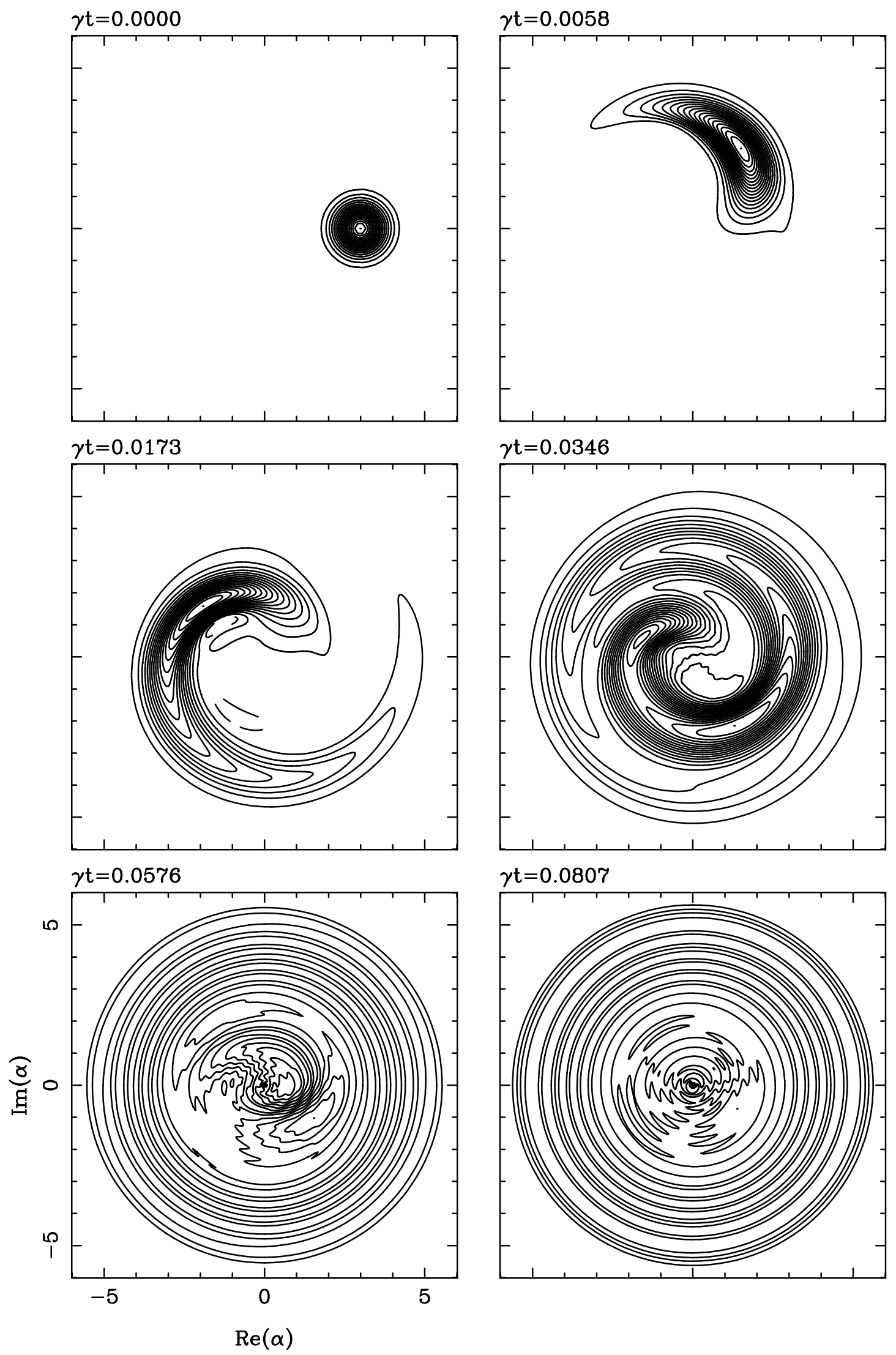

Fig.3 G. M. D'Ariano, M. Fortunato, and P. Tombesi Time evolution of an anharmonic oscillator 

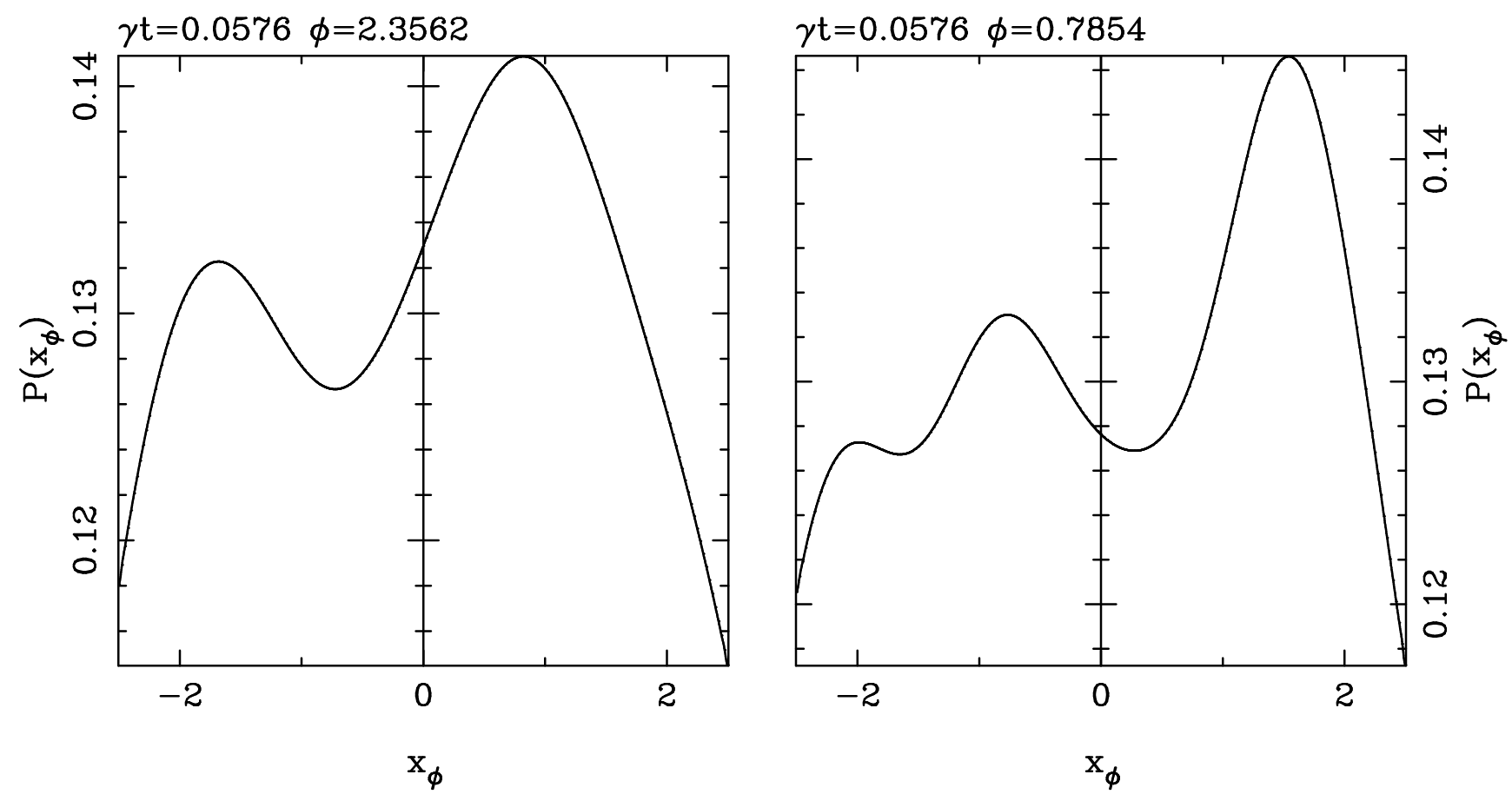

Fig.4 G. M. D'Ariano, M. Fortunato, and P. Tombesi Time evolution of an anharmonic oscillator ... 